\section{Rehabilitación implanto- soportada en un paciente edéntulo con maxilares atróficos. Reporte de caso}

\author{
Implant-supported rehabilitation in edentulous patient with jaw \\ atrophic. Case report
}

\begin{abstract}
Resumen
Uno de los desafíos de la Odontología es la rehabilitación oral de los pacientes totalmente edéntulos con maxilares atróficos. Este caso clínico reporta un protocolo de tratamiento implantológico realizado en una paciente adulto mayor con maxilares edéntulos totales y con atrofia avanzada, en la cual se realizó una rehabilitación implanto-soportada e implanto-retenida consistente en una prótesis híbrida superior apoyada en seis implantes y una sobredentadura inferior retenida por una barra fija conectada a cuatro implantes, con un seguimiento total de un ańo, sin reportar complicaciones. Este caso nos otorga una evidencia de un protocolo implantológico y rehabilitador para pacientes con atrofias severas mejorando así la poca retención y estabilidad que presentan las prótesis convencionales en maxilares atróficos, además de mostrar los beneficios en cuanto a eficacia masticatoria y mejor calidad de vida en pacientes con este diagnóstico.

Palabras clave: Implantes dentales; Prótesis dental; sobredentadura; mandíbula edéntula.
\end{abstract}

\begin{abstract}
One of the challenges of dentistry is oral rehabilitation in totally edentulous patients with atrophic jaws. This case reports a protocol for implant treatment performed in elderly patient with an edentulous jaws and advanced atrophy, in which an implant-retained implant-supported and consistent rehabilitation was conducted in a superior hybrid prosthesis supported by 6 implants and on lower denture retained by a horizontal bar attached to 4 implants, with a total follow-up of 1 year, without reporting complications. This case gives us evidence of an implant and rehabilitation protocol for patients with severe atrophy thus improving poor retention and stability posed by conventional prostheses in atrophic jaws, and show the benefits in terms of masticatory efficiency and better quality of life for patients with this diagnosis.

Keywords: Dental implants; Dental prosthesis; Denture; overlay; Jaw; edentulous.
\end{abstract}

Caso Clínico

\author{
Ivonne Alayo Silva ${ }^{1 a}$, Sixto Grados \\ Pomarino ${ }^{1 \mathrm{a}}$. \\ 1 Facultad de Odontología. Universidad Nacional \\ Mayor de San Marcos. Lima, Perú. \\ a Cirujano Dentista. Especialista en Periodoncia.
}

Correspondencia:

Ivonne Alayo Silva

Correo electrónico: ivonne_dil@hotmail.com

Mza. A Lote 06. Urb. Bruces. Nuevo Chimbote. El Santa.

\section{Coautor:}

Sixto Grados Pomarino

sixto.grados.p@unmsm.edu.pe

Conflicto de intereses: Los autores declaran no tener conflictos de interés.

Fuente de financiamiento: Ninguno.

\section{Introducción}

La ausencia de estímulo posterior a la exodoncia provoca una reducción de las trabéculas en esa zona, disminuyendo así el ancho de hueso y posteriormente en su altura como resultado de los mecanismos de modelado y remodelado óseo 1. Esta atrofia ocurre de manera lenta y continua con los ańos, variando de un individuo a otro, pudiendo ser acelerado por el uso de prótesis totales convencionales. El tratamiento con implantes dentales en pacientes mayores requiere un diagnóstico integral y una planificación de tratamiento para conseguir resultados funcionales y estéticos ${ }^{2}$.
Las prótesis hibridas son fijas para el paciente, pero removibles para el profesional, son de carácter implanto-soportada e implanto-retenida, permiten la posibilidad de una unión directa de la prótesis a los implantes y la transmisión de las fuerzas asegurando no solamente la estabilidad de la prótesis, sino también disminuyendo los traumatismos sobre los tejidos blandos de la boca, favoreciendo la sensación que sus dientes artificiales han llegado a formar parte integral de ellos mismos, lo que aumenta extraordinariamente la calidad de vida de los pacientes ${ }^{3}$.

Se denominan sobredentaduras a las prótesis totales con apoyo de dientes o de implantes. Las sobredentaduras, no sólo consiguen apoyo y retención en las raíces o en los implantes, además en comparación con las prótesis totales, aumentan considerablemente la fuerza y la eficiencia masticatoria. La capacidad sensorial por medio de los receptores periodontales desempeńa un papel muy importante en la función neuromuscular ${ }^{4}$. Para la colocación de una barra en la mandíbula, los implantes deben anclarse en las regiones donde antes se ubicaban los caninos e incisivos laterales, de esta manera se obtiene una barra suficientemente larga y correctamente colocada, las barras muy cortas o excesivamente distales provocan pro- 
blemas funcionales, y las barras diagonales, cargas de torsión ${ }^{5}$. La tendencia al fracaso de implantes en pacientes desdentados se observa cuando no existe un seguimiento periódico, siendo las complicaciones más frecuentes, los desgastes y la fractura de la prótesis, hubo menor cantidad de pérdida ósea en prótesis que tenían mayor número de implantes, según un estudio realizado por Jemt y Johansson ${ }^{6}$. En el presente reporte de caso clínico se describe el tratamiento de un paciente adulto mayor edéntulo total, con atrofia de rebordes maxilares que fue rehabilitado con prótesis implanto soportadas.

\section{Reporte del caso}

Paciente de sexo femenino de 76 ańos de edad acudió a la clínica de posgrado de la Facultad de Odontología de la Universidad Nacional Mayor de San Marcos, Lima, Perú. En el interrogatorio refirió ser portadora de prótesis total por más de 25 años aproximadamente, manifestando dificultad en la función de sus prótesis totales por poseer poca retención y estabilidad, limitándola en su alimentación.

La paciente refirió padecer de triglicéridos elevados, hipertensión arterial controlada con medicamentos, además del diagnóstico de lipoma benigno en piso de boca diagnosticado mediante biopsia, recibiendo control del mismo cada tres meses en el área de cirugía de cabeza y cuello, refirió además estar en tratamiento reumatológico por padecer artrosis, debido al adecuado control de las enfermedades sistémicas de la paciente y previa interconsulta con su médico tratante se procedió a dar inicio al examen clínico extra e intraoral, destacando la marcada atrofia de la arcada edéntula inferior, también se evaluó la dimensión vertical a través del método fonético y de las proporciones faciales evidenciando reducción de la distancia de la dimensión vertical así como un perfil convexo con marcada protusión mandibular. El examen radiológico reveló la escasa disponibilidad ósea, debido a la marcada atrofia en ambos maxilares (Figura 1).

El diagnóstico fue edéntulo superior Tipo 3 división B D C y edéntulo inferior Tipo 2 división A C, según la clasificación de Kent ${ }^{7}$ y la universidad de Luisiana con divisiones de Mish y Judy 7 , en la clasificación sub antral de Misch ${ }^{8}$ presentó clase SA-3 y atrofia maxilar grupo II, como atrofia mandibular grupo III de Cawood y Howel ${ }^{9}$.
Luego de realizar los cortes tomográficos correspondiente ubicando las áreas con mayor disponibilidad ósea, se realizó una planificación protésica e implantológica conveniente para este caso: Una prótesis híbrida PF-3 (Prótesis fija clase 3) según la clasificación de Misch para prótesis fijas sobre implantes, ubicada en el maxilar apoyada sobre seis implantes. En la mandíbula se planificó una sobredentadura PR-4 (Prótesis removible clase 4) ${ }^{7}$ apoyada en una barra conectada a cuatro implantes colocados entre los agujeros mentonianos.

El procedimiento quirúrgico realizado en el maxilar consistió en anestesia infiltrativa con Scandicaine al $2 \%{ }^{\circledR}$ (mepivacaína + adrenalina), se procedió a realizar las incisiones correspondientes a las áreas a implantar, decolado a espesor total, colocación de la guía quirúrgica, empezando la perforación de cortical con fresa lanza, luego se completó la secuencia de fresado y verificación con pin de paralelismo. Se procedió a la inserción de los implantes modelo Seven de marca Mis Implant (Israel MIS $\left.{ }^{\circledR}\right)$, dos implantes fueron colocados en zona de tuberosidad bilaterales donde se contaba con aproximadamente 6 $\mathrm{mm}$ de altura según la tomografía, por lo cual se realizó elevación del piso de seno maxilar con la técnica de Summers elevando $4 \mathrm{~mm}$ y colocación simultánea de dos implantes de diámetro 3,75 x $10 \mathrm{~mm}$ de longitud, dos implantes en zona premolar bilateral de diámetro $3,75 \times 11,5 \mathrm{~mm}$ de longitud, en la zona de canino e incisivo lateral derecha se colocó un implante de diámetro estrecho 3,30 x $10 \mathrm{~mm}$ de longitud debido a la falta de anchura ósea, en la zona canina izquierda se instaló un implante de diámetro $3,75 \times 10 \mathrm{~mm}$ de longitud, todos los implantes alcanzaron buena estabilidad primaria y torque de 35 a $40 \mathrm{~N}$, luego se realizó la colocación de respectivas tapas de cierre, la síntesis del colgajo consistió en puntos simples independientes, realizados con ácido poliglicólico 4/0. Se recetó amoxicilina 500 mg, 18 tabletas, además de diclofenaco sódico de $50 \mathrm{mg}$ más paracetamol de $500 \mathrm{mg} 15$ tabletas, indicándose una tableta de cada medicamento cada 8 horas por 5 días También se indicó dieta blanda, y reposo. Se citó para control post operatorio en 72 horas.

El procedimiento quirúrgico en la mandíbula consistió en anestesia infiltrativa de Scandicaine al $2 \%{ }^{\oplus}$ (mepivacaína + adrenalina), se procedió a realizar la incisión supracrestal, el decolado a espesor total fue lo más conservador posible, expuesto el hueso se posicionó la guía quirúrgica y se procedió a realizar las perforaciones con fresa lanza , debido al decolado conservador y a la presencia cercana del nervio dentario inferior el cual según la referencia tomográfica estaba ubicado muy cerca a la cima del reborde debido a la atrofia severa presente, se decidió solo preparar tres lechos quirúrgicos para recibir tres implantes modelo Seven de marca Mis implant ${ }^{\oplus}$ de diámetro 3,75 x 10 mm de longitud. Se realizó la colocación de la tapa de cierre de los implantes y sutura de puntos independientes con ácido poliglicólico 4/0.

En el control radiográfico (Figura 2), se observó los implantes aparentemente paralelos, además se visualizó el área disponible para colocación del implante faltante además de tener una referencia precisa de la lejanía del conductor dentario inferior por lo cual se realizó una tercera intervención para insertar el cuarto implante de modelo Seven de 3,75 mm de diámetro x $10 \mathrm{~mm}$ de largo.

Luego de seis meses posterior a la cirugía implantológica se realizó la colocación de los cicatrízales que fueron rec-

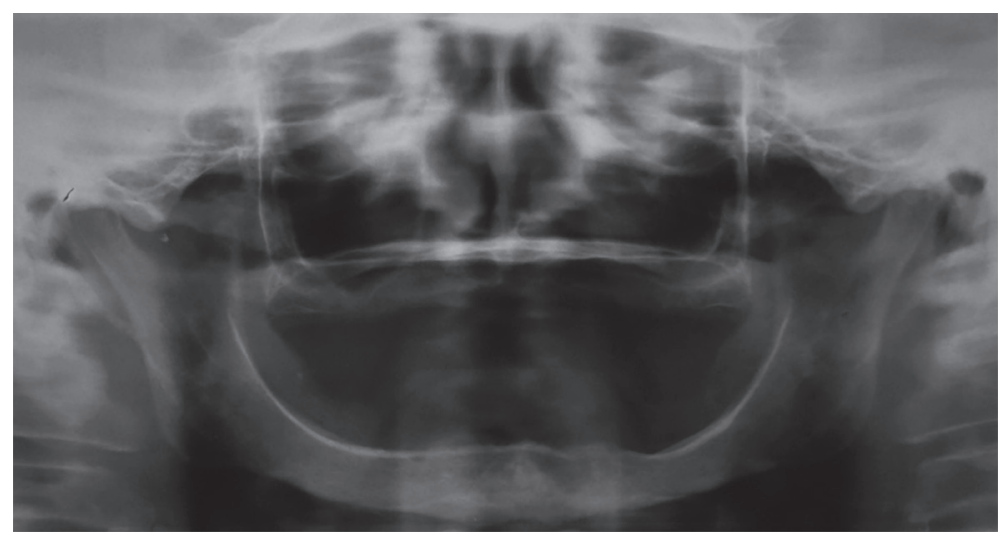

Figura 1. Radiografía panorámica previa. Se evidencia la marcada atrofia de los maxilares 
tos y con un transepitelial de $3 \mathrm{~mm}$; la técnica excisional del punch, para retirar tejido blando sobre los implantes, fue escogida para colocar los cicatrízales. Luego de dos semanas se realizó la impresión con cubeta individual y técnica de cubeta abierta utilizando silicona (Zetaplus) de condensación. Se obtuvo el modelo de trabajo y se realizó los encerados de las futuras prótesis, las cuales fueron instaladas en el paciente para realizar las pruebas respectivas (Figura 3). Se instaló la prótesis híbrida acrílica maxilar, verificándose de manera radiográfica la adecuada adaptación de la conexión entre los pilares y la plataforma de los implantes, se procedió a aplicar un torque de $25 \mathrm{~N}$ a cada pilar, además se instaló la barra mandibular de igual manera se procedió a verificar el asentamiento de los pilares en las plataformas de los implantes, una vez conforme se realizó un torque de $25 \mathrm{~N}$ a cada pilar (Figura 4), finalmente se posicionó la sobredentadura y se procedió a controlar la oclusión del paciente programándose los controles respectivos. La paciente fue incluida en un tratamiento de mantenimiento y control cada tres meses que incluyó el refuerzo de la educación y motivación sobre el control del biofilm dental, cepillado e higiene de las

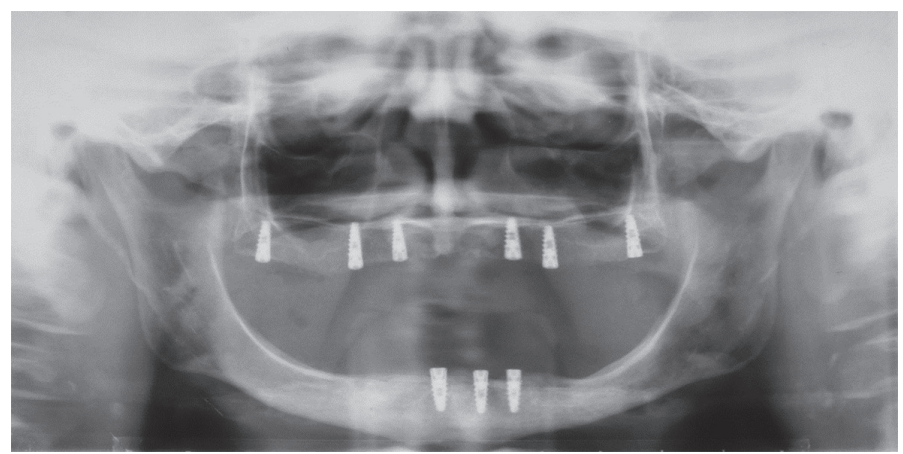

Figura 2. Radiografía post operatoria. Colocación de los implantes maxilares y mandibulares. El cuarto implante maxilar fue colocado posterior a la toma radiográfica

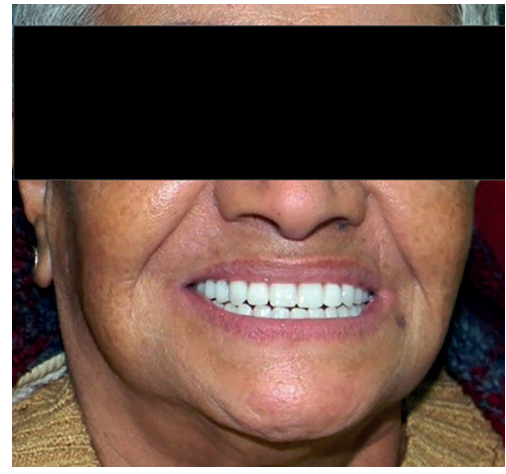

Figura 3. Prueba de prótesis enceradas. Se evidencia apropiada exposición de dientes prótesis dentales. Después de un periodo de seguimiento de un ańo no se han reportado complicaciones (Figura 5).

\section{Discusión}

La rehabilitación de edéntulos totales con implantes dentales son una alternativa predecible desde el primer estudio reportado por Branemark en $1977^{10}$.

Maló en el año 2003, publicó el primer estudio donde propone el protocolo "All on four" ("Rehabilitación sobre cuatro implantes") realizado inicialmente con el Sistema Branemark 11, posteriormente dos años más tarde con el Sistema Nobel ${ }^{12}$, ambos estudios demostraron altas tasas de supervivencia de los implantes (98,2\% y $97,6 \%$ respectivamente). Estos estudios concluyen que la realización de prótesis fijas sobre cuatro implantes es un tratamiento predecible tanto en el maxilar como en la mandíbula, estos datos nos indican que una prótesis fija sobre seis implantes sería un tratamiento con alta tasa de supervivencia al contar con un mayor número de implantes para distribuir la carga masticatoria de manera más equitativa y evitando los cantilevers distales.
Buscando superar limitaciones propias de las atrofias maxilares, se han realizado estudios donde se propone un protocolo simplificado, consistente en la colocación de implantes inclinados que soporten una prótesis fija, este estudio fue realizado por Calandriello y Tomati en el $2005^{13}$, obteniendo una tasa de supervivencia de $96 \%$ de los implantes ${ }^{7}$, posteriormente en el 2007 Capelli y cols. ${ }^{14}$, realizaron un estudio que evaluó la supervivencia en implantes verticales, en comparación con implantes inclinados, obteniendo tasas de supervivencia similares de $97,8 \%$ y $97,2 \%$ respectivamente, estos estudios sugieren que en el presente reporte de caso también se pudo considerar la posibilidad de realizar implantes inclinados, con lo cual se hubiese podido colocar implantes de mayor longitud, sin embargo hubiera traído como consecuencias el uso de pilares intermedios que permitan la adaptación de la prótesis fija, lo cual tendría como desventaja el aumento del costo del tratamiento, valorando el factor costo beneficio para el paciente se realizó la colocación de implantes verticales obteniendo buenos resultados y una prótesis a menor costo.

Otro factor importante en la tasa de éxito y supervivencia de las prótesis fijas es el periodo de seguimiento, así se demostró en un estudio realizado por Jemt y Johansson en el $2006^{6}$, donde se evaluó por un periodo de 15 años la tasa de supervivencia de los implantes que soportaban una prótesis fija maxilar, siendo este de 90,6\%, el fracaso de implantes estuvo relacionado con periodos inexistentes de seguimiento y se observó que a mayor número de implantes se encontró menor pérdida ósea, hecho que sería controversial con respecto al protocolo "all on four" que reduce el número de implantes a solo cuatro para sostener una prótesis fija, en el caso clínico desarrollado no fue posible colocar un número mayor a seis implantes debido a la marcada atrofia presente. Sin embargo, en contraste

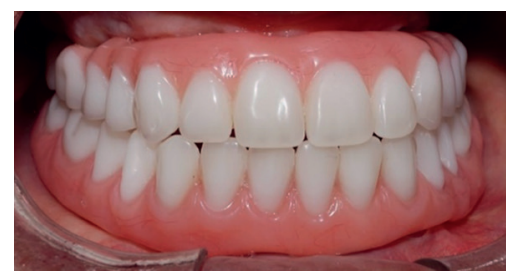

Figura 5. Fotografía al ańo de seguimiento. No se evidencia complicaciones de tejidos blandos ni de tejidos duros 
con este estudio un año después Maló y cols. ${ }^{15}$ aplicaron una técnica sin colgajo y guiada por el ordenador, obteniendo una tasa de supervivencia de 97,8\%, la cual está por encima del resultado encontrado por Jemt y Johanson ${ }^{6}$, sin embargo, debemos recordar que Maló ${ }^{16,17}$ solo reportó un periodo de seguimiento de uno a cinco ańos, a diferencia de Jemt y Johansson ${ }^{6}$ quienes reportaron un seguimiento de 15 años, lo cual sería un factor determinante en la diferencia de ambas tasas.

Capelli y cols. ${ }^{14}$, evaluaron la colocación de implantes dentales inclinados en maxilares atróficos obteniendo como resultado una tasa de supervivencia de $96 \%$, la reabsorción ósea media fue de $0,34 \mathrm{~mm}$, sugiriendo que los implantes inclinados en maxilares atróficos simplifican los procedimientos de tratamiento; sin embargo, en el presente caso clínico la colocación de los implantes fue vertical a pesar de ser realizada en maxilares atróficos teniendo una tasa de supervivencia de 100\% sin ninguna complicación.

\section{Conclusiones}

La colocación de implantes en maxilares con rebordes atróficos es un tratamiento predecible si se realiza un adecuado diagnóstico y se desarrolla un meticuloso plan de tratamiento.

La realización de una prótesis implanto soportada en el maxilar superior colocada sobre seis implantes fue un tratamiento con alta tasa de éxito y sin complicaciones reportadas luego de 12 meses de seguimiento.

La rehabilitación completa sobre implantes proporcionó al paciente estabilidad y retención de ambas prótesis, restableciendo así la función masticatoria del desdentado total, recuperando confort y estética, mejorando así su calidad de vida e incrementado la autoestima y seguridad perdida por la inestabilidad de sus prótesis convencionales.

\section{Referencias bibliográficas}

1. Misch C. Implantología Contemporánea. $1^{\mathrm{a}}$ ed. Madrid: Mosby Doyma; 1995.

2. Baladron J. Cirugía Avanzada en Implantes.1 $1^{a}$ ed. Espańa: Atlante; 2000.

3. Mericske-Stern R, Worni A. Optimal number of oral implants for fixed reconstructions: a review of the literature. Eur J Oral Implantol. 2014;7(2):133-153.

4. Dudley J. Maxillary implant overdentures: current controversies. Aust Dent J. 2013;58:420-3.

5. Zou D, Wu Y, Huang W, Wang F, Wang S, Zhang Z. A 3-year prospective clinical study of telescopic crown, bar, and locator attachments for removable four implantsupported maxillary overdentures. Int J Prosthodont. 2013;2:566-73.

6. Jemt $T$, Johansson J. Implant treatment in the edentulous maxillae: A 15-year follow-up study on 76 consecutive patients provided with pixed Prostheses. Clin Implant Dent Relat Res. 2006;8(2):61-69.

7. Misch C. Contemporany Implant Dentistry. 3 ed. EEUU: Mosby Elsevier; 2008.

8. Hunt D, Jovanovic S. Autogenous bone harversting: a chin graft technique for particulate and monocortical bone blocks. Int J Periodontics Restorative Dent. 1999;19(2):165-73.

9. Cawood JI, Howell RA. A classification of the edentulous jaws. Int J Oral Maxillofac Surg. 1988;17(4):232-6.

10. Velasco E, Pato J, García A, Lorrio JM, Cruz JM. El tratamiento con implantes en pacientes edéntulos totales mandibulares mediante rehabilitación fija. Av Periodon Implantol. 2007;19(3):151-159.
11. Maló P, Rangert B, Nobre M. "Allon-Four" inmediate-function concept with Branemark system implants for completely edentulous mandibles: a retrospective clinical study. Clin Implant Dent Relat Res. 2003;5(1):2-9.

12. Maló P, Rangert B, Nobre M. Allon-4 immediate-function concept with Brånemark System implants for completely edentulous maxillae: a 1-year retrospective clinical study. Clin Implant Dent Relat Res. 2005;7(1):88-94.

13. Calandriello R, Tomatis M. Simplified treatment of the atrophic posterior maxilla via immediate/ early function and tilted implants: a prospective 1-year clinical study. Clin Implant Dent Relat Res. 2005;7(S1):1-12.

14. Capelli M, Zuffetti F, Del Fabbro $\mathrm{M}$, Testori T. Immediate rehabilitation of the completely edentulous jaw with fixed prostheses supported by either upright or tilted implants: a multicenter clinical study. Int J Oral Maxillofac Implants. 2007;22(4):639-44.

15. Maló P, de Araujo Nobre M, Lopes A. The use of computerguided flapless implant surgery and four implants placed in immediate function to support a fixed denture: preliminary results after a mean follow-up period of thirteen months. J Prosthet Dent. 2007;97(6):S26-34.

16. Maló P, Araújo M, Lopes A, Rodrigues R. Double full-arch versus single full-arch, four implant-supported rehabilitations: a retrospective, 5-year cohort study. J Prosthodont. 2015;24(4):263-70.

17. Maló P, Araújo M, Lopes A, Rodrigues R. Immediate loading short implants çinserted on low bone quantity for the rehabilitation of the edentulous maxilla using an all-on-4 design. J Oral Rehabil. 2015;42(8):615-23. 